\title{
Tomografia Eletromagnética de Tempos de Trânsito: Aplicação do GCV para a Busca do Parâmetro de Regularização
}

\author{
Electromagnetic Traveltime Tomography \\ GCV Application for the Search of the Regularization Parameter
}

Naiane Pereira de Oliveira*

Instituto de Geociências - UFBA

Amin Bassre计

Instituto de Geociências - UFBA

Centro de Pesquisa em Geofísica e Geologia - UFBA

Instituto Nacional de Ciência e Tecnologia de Geofísica de Petróleo

Campus Universitário de Ondina

Salvador - BA - 40210-340

\begin{abstract}
Na inversão tomográfica eletromagnética com a geometria de aquisição interpoços utilizamos como dados de entrada, o tempo de trânsito do pulso eletromagnético de alta frequência transmitido de uma antena à outra, desconsiderando a forma da onda em si. A inversão tomográfica eletromagnética é mal-condicionada, tal como a maioria dos problemas inversos em Geofísica de Exploração. Por esse motivo, recorremos à regularização por matrizes de derivadas como ferramenta matemática na obtenção de uma solução factível. Essa abordagem possui uma constante chamada de parâmetro de regularização $(\lambda)$, cuja escolha também constitui um problema. Para a seleção desta constante usamos uma técnica conhecida como Validação Cruzada Generalizada, que é um método baseado em considerações estatísticas. Os modelos recuperados apresentam boa concordância com o respectivo modelo verdadeiro, comprovando a aplicabilidade da tomografia eletromagnética no imageamento de reservatórios geológicos.
\end{abstract}

Tomografia Eletromagnética; Inversão; Regularização; Caracterização de Reservatórios; GCV.

The electromagnetic tomographic inversion in crosswell geometry uses the transit time of the electromagnetic pulse of high frequency transmitted from one antenna to another as input data, disregard the waveform. The electromagnetic tomographic inversion is ill-posed, as the most of inverse problems in geophysics exploration. For this reason, we have used the regularization by derived matrices as mathematical tool in obtaining a feasible solution. This approach has a constant called regularization parameter $(\lambda)$, whose choice is also a problem. To select this constant we have used a technique known as Generalized Cross Validation, which is a method based on statistical considerations. Recovered models show good agreement with their true model, testifying the applicability of electromagnetic tomography in the imaging of geological reservoirs.

Key-words: Electromagnetic Tomography; Inversion; Regularization; Reservoir Characterization; GCV.

\section{INTRODUÇÃ̃O}

\footnotetext{
*Endereço Eletrônico: naipereiraoliveira@gmail.com

${ }^{\dagger}$ Endereço Eletrônico: bassrei@ufba.br
}

A tomografia foi incorporada na Geofísica de Exploração de hidrocarbonetos como método de inversão de dados gerando modelos de velocidade da subsuperfície. Na metodologia de inversão tomográfica eletromagnética em geometria interpoços (cross-well) utilizamos o 
tempo de trânsito do pulso eletromagnético de alta frequência transmitido de uma antena à outra como dados de entrada (d), desconsiderando a forma da onda. A velocidade de propagação do pulso depende das propriedades eletromagnéticas intrínsecas do meio, ou seja, a condição de propagação é determinada pela permeabilidade magnética $\mu$ e pela permissividade elétrica $\epsilon$.

A inversão tomográfica é classificada como um problema inverso mal-posto, devido ao fato das condições de existência, unicidade e estabilidade não serem completamente satisfeitas. Nesse sentido, algoritmos iterativos de resolução de sistemas lineares com regularização possuem o compromisso de fornecer soluções fidedignas.

\section{PROBLEMAS INVERSOS E DECOMPOSIÇÃO POR VALORES SINGULARES}

A inversão é a técnica empregada em problemas que estimam os parâmetros de um determinado modelo utilizando os dados medidos como dados de entrada. De forma contrária, a técnica de modelagem direta resulta em criar um modelo sintético para a obtenção dos referidos dados. No processo de resolução do problema inverso, deve-se realizar uma análise prévia com relação às questões de existência, unicidade e estabilidade da solução, se uma dessas condições falha o problema é dito malposto [1].

A inversa do operador matricial $\mathbf{G}$ é a pseudo-inversa generalizada de Moore-Penrose $\mathbf{G}^{+}$. O método da Decomposição por Valores Singulares ou SVD, do inglês Singular Value Decomposition, consiste numa técnica de obtenção da pseudo-inversa de uma matriz G onde a matriz original é decomposta, supondo ser uma matriz retangular $M \times N$ de posto $k$, em valores singulares tal como:

$$
\mathbf{G}=\mathbf{U} \mathbf{\Sigma} \mathbf{V}^{T},
$$

onde $\mathbf{U}$ é a matriz $M \times M$ que contém os autovalores ortonormalizados de $\mathbf{G G}^{T}, \mathbf{V}$ é a ma- triz $N \times N$ que contém os autovalores ortonormalizados de $\mathbf{G}^{T} \mathbf{G}$ e $\boldsymbol{\Sigma}$ é a matriz diagonal que contém os valores singulares de $\mathbf{G}$ colocados em ordem decrescente. A inversa de MoorePenrose é definida por uma matriz dada por:

$$
\mathbf{G}^{+}=\mathbf{V} \boldsymbol{\Sigma}^{+} \mathbf{U}^{T}
$$

onde $\boldsymbol{\Sigma}^{+}$representa a matriz diagonal com os valores recíprocos dos valores singulares nãonulos de $\boldsymbol{\Sigma}$.

\section{REGULARIZAÇÃO E O MÉTODO GCV}

O emprego da técnica SVD está, via de regra, associada à existência dos pequenos valores singulares, que quando utilizados na construção da matriz inversa, implicam no comprometimento da qualidade da solução. Alguns critérios podem ser utilizados para se contornar o problema dos pequenos valores singulares como abordado em [2].

Neste trabalho optamos por técnicas de regularização que são empregadas com o intuito de tornar a inversão mais estável. No entanto, ambos os critérios podem ser utilizados, por exemplo em [3] onde através de um algoritmo de inversão utilizou-se o SVD com seleção de valores singulares e regularização.

As técnicas de regularização permitem solucionar não o problema original, mas um problema equivalente e mais robusto com relação às variações nos dados de entrada. A solução pela regularização por matrizes de derivadas, obtida minimizando a função objetivo $\Phi(\mathbf{m})$, é definida como sendo [4]:

$$
\Phi(\mathbf{m})=\mathbf{e}^{T} \mathbf{e}+\lambda \mathbf{m} \mathbf{D}_{n}^{T} \mathbf{D}_{n} \mathbf{m},
$$

onde $\mathbf{e}=\mathbf{d}-\mathbf{G m}$. No segundo termo, $\mathbf{D}_{n}$ é normalmente a discretização de um operador de derivadas impondo alguma suavização na solução, $\lambda$ é uma constante, $\lambda>0$, chamada de parâmetro de regularização que controla a quantidade de suavização sobre a solução. Para o caso em que $\lambda=0$ a regularização não é realizada e a inversão se reduz aos Métodos dos 
Mínimos Quadrados.

Resolvendo a Eq. (3), obtemos:

$$
\mathbf{m}^{e s t}=\left(\mathbf{G}^{T} \mathbf{G}+\lambda \mathbf{D}_{n}^{T} \mathbf{D}_{n}\right)^{+} \mathbf{G}^{T} \mathbf{d}^{o b s} .
$$

Quando $n=0, \mathbf{D}_{n}$ corresponde à matriz identidade resultando em uma regularização de ordem zero e a solução se reduz ao Método dos Mínimos Quadrados Amortecidos. Para $n=1$, $\mathbf{D}_{n}$ corresponde à matriz de primeira derivada e a regularização é de primeira ordem. Quando $n=2$ temos a regularização de ordem dois que culmina no uso da matriz da aproximação numérica da segunda derivada.

A escolha do parâmetro de regularização $(\lambda)$ é de crucial importância na busca de soluções mais realistas. Na literatura existem várias técnicas empregadas na escolha de $\lambda$, tais como a curva $L$ e a curva $\Theta$ ambas ferramentas gráficas utilizadas de forma analítica para escolha do parâmetro de regularização, usualmente aplicada em problemas inversos em Geofísica como pode ser verificado nos trabalhos [5] 8 .

Escolhemos uma técnica que sido utilizada por outros pesquisadores para a seleção do parâmetro de regularização baseada em considerações estatísticas, chamada de validação cruzada generalizada, ou GCV, do inglês Generalized Cross Validation. A GCV sugere que um bom valor para o parâmetro de regularização deve prever dados do vetor de tempos de trânsito que estejam faltando ou que foram retirados. Mais precisamente, se um elemento do vetor de dados for retirado, então a solução regularizada correspondente deve prever bem essa falta. Baseado nesse princípio, o parâmetro de regularização é o valor de $\lambda$ que minimiza a função GCV [9]:

$$
G C V(\lambda)=\frac{\left\|\mathbf{d}^{o b s}-\mathbf{d}\left(\mathbf{m}_{\lambda}\right)\right\|^{2}}{\{\operatorname{tr}[I-A(\lambda)]\}^{2}},
$$

sendo $\mathbf{A}(\lambda)=\mathbf{G}\left(\mathbf{G}^{T} \mathbf{G}+\lambda \mathbf{D}_{n}^{T} \mathbf{D}_{n}\right)^{+} \mathbf{G}^{T}$. Na comunidade geofísica, o critério GCV foi aplicado na inversão 1-D de dados eletromagnéticos por C.G. Farquharson e D.W. Oldenburg [10], em tomografia de difração por E.T.F. Santos e A. Bassrei [11] e C.J.M.G. Silva [12], e em tomo- grafia de tempos de trânsito por N.P. Oliveira e A. Bassrei [13].

\section{MÉTODO DA INVERSÃO LINEARIZADA}

Neste método parte-se de um modelo inicial, $\mathbf{m}_{0}$, que é reformulado sucessivamente, também conhecido como método de GaussNewton que lineariza o problema não linear $g(\mathbf{m})=\mathbf{d}$, em torno de uma solução aproximada. Então, a estimativa do modelo é obtida de forma iterativa resolvendo-se um sistema de equações lineares a cada passo do processo iterativo. O método convergirá para o modelo associado ao mínimo da função custo mais próoximo de $\Phi\left(\mathbf{m}_{0}\right)$ [1. Sendo assim, a expressão para atualizar o modelo corrente é:

$\mathbf{m}^{k+1}=\left[\left(\mathbf{G}^{T} \mathbf{G}+\lambda \mathbf{D}_{n}^{T} \mathbf{D}_{n}\right)^{k}\right]^{+}\left(\mathbf{G}^{T}\right)^{k} \Delta \mathbf{d}^{k}+\mathbf{m}^{k}$,

onde: $\left[\left(\mathbf{G}^{T} \mathbf{G}+\lambda \mathbf{D}_{n}^{T} \mathbf{D}_{n}\right)^{k}\right]^{+}$a pseudo-inversa da matriz $\left(\mathbf{G}^{T} \mathbf{G}+\lambda \mathbf{D}_{n}^{T} \mathbf{D}_{n}\right)^{k}$ na $k$-ésima iteração; $\Delta \mathbf{d}^{k}$ é a diferença entre os dados da iteração $k+1$ e os dados da $k$-ésima iteração; $\mathbf{m}^{k}$ são os parâmetros atuais do modelo na $k$-ésima iteração; $\mathbf{m}^{k+1}$ são os parâmetros estimados para a iteração $k+1$.

\section{TOMORAFIA ELETROMAGNÉTICA DE TEMPOS DE TRÂNSITO}

A tomografia é uma técnica de reconstrução de imagens com muitas aplicações em Geofísica [14. A tomografia é baseada na ideia de que um conjunto de dados observados consiste de integrais ao longo de linhas de alguma quantidade física:

$$
t_{i}=\int_{R_{i}} s(x, z) d l=g[s(x, z)],
$$

sendo $t_{i}$ o tempo de trânsito para o $i$-ésimo raio; $R_{i}$ o raio ao longo do qual é realizada a integração; $d l$ o elemento de raio; $s(x, z)$ a vagarosidade do meio no ponto $(x, z)$, em que 
$x$ é a coordenada horizontal e $z$ é a coordenada vertical e $g[s(x, z)]$ representa a variável $g$ em função da vagarosidade. A Eq. (7) tem um comportamento não linear por descrever o percurso do raio em meios não homogêneos e anisotrópicos.

Uma maneira de parametrização mais simples para uma distribuição de velocidades é dividir a região de estudo em pequenas células ou blocos e atribuir valores constantes à vagarosidade (recíproco da velocidade) em cada célula. Por sua vez, a propagação da onda pode ser modelada através de modelagens analíticas ou numéricas.

$\mathrm{Na}$ tomografia eletromagnética interpoços o pulso eletromagnético de alta frequência é transmitido por uma antena em um poço e recebido por uma antena em outro poço. A velocidade de propagação do pulso depende das propriedades elétricas intrínsecas do meio, ou seja, a condição de propagação é determinada pelas propriedades eletromagnéticas: a permeabilidade magnética $\mu$ e a permissividade elétrica $\epsilon$. A permeabilidade magnética é uma propriedade dos meios materiais que influi no comportamento da propagação da onda eletromagnética, porque mede a facilidade com que um corpo se magnetiza sobre a ação de um campo magnético externo. Expressa como $\mu=\mu_{0}\left(1+\chi_{m}\right)$, onde $\mu_{0}$ é a permeabilidade magnética do vácuo $\left(\mu_{0}=4 \pi \times 10^{-7}\right.$ $\mathrm{H} / \mathrm{m})$ e $\chi_{m}$ é a suscetibilidade magnética. De acordo com a referência [15] a permeabilidade relativa $\left(\mu_{r}=\mu / \mu_{0}\right)$ raramente é apreciavelmente maior que a unidade, com exceção para poucos minerais magnéticos. Significa que o parâmetro $\chi_{m}$ é aproximadamente igual a zero, ou $\mu \approx \mu_{0}$. Deste modo, variações nas velocidades devem ser devidas às permissividades elétricas $(\epsilon)$. Essa grandeza representa a facilidade com que um meio semi-condutor se polariza sobre a ação de campos elétricos externos. Essa polarização pode ser eletrônica, iônica ou molecular. Desta forma, temos para a velocidade a relação:

$$
v=\frac{c}{\sqrt{\epsilon_{r}}}
$$

sendo $\epsilon_{r}$ a constante dielétrica e $c$ a velocidade da onda eletromagnética no vácuo. A constante dielétrica é definida pela razão $\epsilon_{r}=\epsilon \epsilon_{0}$, onde $\epsilon_{0}$ é a permissividade elétrica no vácuo $\left(\epsilon_{0}=8,854 \times 10^{-12} \mathrm{~F} / \mathrm{m}\right)$.

\section{TRAÇADO DO RAIO}

Uma maneira mais lógica de analisar o traçado de raios sem envolver a conceito de frente de onda é pelo princípio de Fermat. Vários métodos estão descritos na literatura para determinar o caminho descrito pelo raio entre dois pontos. A seguir é descrito o algoritmo numérico proposto por A.H. Andersen e A.C. Kak [16]. Aplicando o princípio de Fermat e sabendo que a equação de Euler constitui uma condição necessária para a existência de um valor extremo da integral $\int_{P_{1}}^{P_{2}} n d s$, obtemos a seguinte equação diferencial para um meio heterogêneo:

$$
\frac{d}{d s}\left(n \frac{d \mathbf{r}}{d s}\right)=\nabla n
$$

onde $n(x, z)$ é o índice de refração na posição $(x, z) ; \mathbf{r}$ é o vetor posição do raio; $d \mathbf{r} / d s$ é o vetor tangente ao raio em $(x, z)$; $d s$ é o elemento de comprimento de trajetória de raio; $\nabla n \equiv d n / d \mathbf{r}$ é o gradiente do índice de refração $n(x, z)$. Esta equação diferencial é denominada como equação do raio e, para uma certa vizinhança regular (aquela em que o índice de refração varia suavemente), sua solução representa uma família de raios de menor comprimento acústico [16].

Desenvolvendo a equação do raio, obtém-se:

$$
\left(\nabla n \cdot \frac{d \mathbf{r}}{d s}\right) \frac{d \mathbf{r}}{d s}+n \frac{d^{2} \mathbf{r}}{d s^{2}}=\nabla n
$$

Expandindo o vetor posição em série de Taylor no ponto $s+\Delta s$, onde $\Delta s$ é o incremento do raio, e considerando somente os três primeiros termos:

$$
\mathbf{r}(s+\Delta s)=\mathbf{r}(s)+\frac{d \mathbf{r}}{d s} \Delta s+\frac{1}{2} \frac{d^{2} \mathbf{r}}{d s^{2}}(\Delta s)^{2} .
$$

Isolando o vetor curvatura $d^{2} \mathbf{r} / d s^{2}$ da Eq. 10 
e substituindo em (11), resulta na seguinte expressão:

$$
\begin{aligned}
\mathbf{r}(s+\Delta s) & =\mathbf{r}(s)+\frac{d \mathbf{r}}{d s} \Delta s+\frac{1}{2 n}[\nabla n \\
& \left.-\left(\nabla n \cdot \frac{d \mathbf{r}}{d s}\right) \frac{d \mathbf{r}}{d s}\right](\Delta s)^{2} .
\end{aligned}
$$

O ponto seguinte do raio é estimado pelas equações:

$$
\begin{aligned}
x_{k+1}= & x_{k}+\cos \alpha_{k} \Delta s \\
& +\frac{1}{2 s_{k}}\left(s_{k, x}-d_{k} \cos \alpha_{k}\right) \Delta s^{2}, \\
z_{k+1}= & z_{k}+\sin \alpha_{k} \Delta s \\
& +\frac{1}{2 s_{k}}\left(s_{k, z}-d_{k} \sin \alpha_{k}\right) \Delta s^{2},
\end{aligned}
$$

sendo que $s_{k, x}$ e $s_{k, z}$ são a vagarosidade, recíproco da velocidade $v_{k}$, na direção $x \mathrm{e} z$, respectivamente. $\mathrm{E} d_{k}$ definido como:

$$
d_{k}=s_{k, x} \cos \alpha_{k}+s_{k, z} \sin \alpha_{k}
$$

Partindo-se de um determinado ponto inicial $\left(x_{0}, z_{0}\right)$, que corresponde à posição da fonte, pode-se obter os sucessivos pontos do raio uma vez que os valores de $\sin \alpha_{k}$ e $\cos \alpha_{k}$ são facilmente calculados. Segundo [16, este método tem algumas limitações no sentido de que erros causados na discretização ou transições bruscas de velocidade podem se tornar acumulativos. Para minimizar este problema, deve-se adotar uma malha com resolução suficiente para que o meio seja amostrado adequadamente, resultando em transições mais suaves de velocidade. Também podem ser utilizadas suavização do campo de velocidades e interpolação bilinear dos índices de refração e de suas derivadas parciais respectivas [16].

Um problema do traçado de raio consiste em encontrar as coordenadas do receptor através do raio que parte da fonte. Para um meio homogêneo, essa ligação fonte-receptor configura um raio reto tornando o problema trivial, porém considerando um meio heterogêneo deve-se levar em consideração a curvatura agregada ao raio, neste caso o problema se resume em predizer o ângulo de lançamento do raio da fonte para que alcance um determinado recep- tor. O processo de ligação entre fonte e receptor é denominado de ray linking. Neste trabalho, a metodologia adotada para o traçado de raios foi a mesma descrita por H.A. Schots [17] que implementou computacionalmente em linguagem Fortran, com o seguinte procedimento:

(i) Calculam-se o ângulo e o tempo de trânsito para cada raio, utilizando-se o linking method, tendo como partida o ângulo entre a linha reta que liga a fonte ao receptor e a horizontal. Existindo uma forte variação na distribuição de vagarosidades, vários raios podem não alcançar os objetivos após $N$ iterações. Neste caso, após o traçado de todos os raios, utiliza-se como partida para os raios não convergentes os ângulos dos raios mais próximos que tenham convergido.

(ii) Utilizando o shooting method e considerando um círculo de raio dado, com centro de coordenadas do receptor $\left(x_{g}, z_{g}\right)$, qualquer raio que cruzar a área e terminar neste domínio pertencerá a este receptor. Utiliza-se o método Newton-Raphson para encontrar o receptor, comparando-se o tempo de trânsito deste raio com o tempo dado em $(i)$ ou de um raio anterior. O raio considerado será aquele de menor tempo, obedecendo ao princípio de Fermat.

\section{METODOLOGIAS E RESULTADOS}

Utilizamos um modelo sintético que representa uma situação geológica singular para analisar a metodologia linearizada empregada, limitado lateralmente por poços com 30 fontes num poço e 30 receptores no outro pocco, de modo a se ter 900 raios ou 900 equações. A discretização do modelo se deu com 800 blocos quadrados com vagarosidade (recíproco da velocidade) constante em cada bloco, sendo 20 blocos na horizontal e 40 blocos na vertical. 


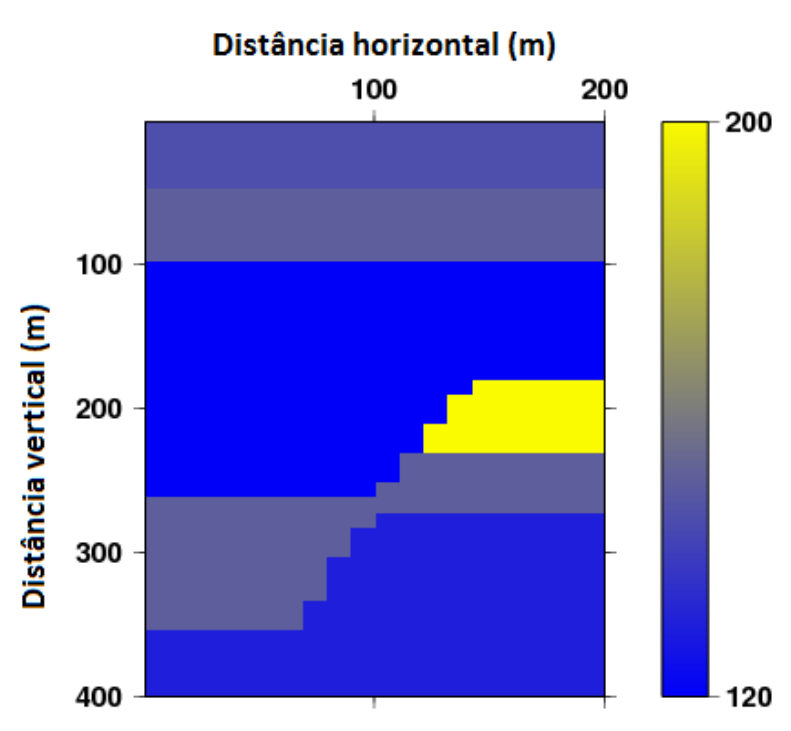

FIGURA 1: Modelo verdadeiro: tomograma eletromagnético. A barra de cores indica as velocidades em $\mathrm{m} / \mu \mathrm{s}$.

A Figura (1) representa tal modelo que, por sua vez, possui 5 regiões de velocidades diferentes com uma variação que vai de 120 a $200 \mathrm{~m} / \mu \mathrm{s}$. Os diferentes valores de velocidade para as rochas estão associados às propriedades elétricas do material rochoso, ou seja, os valores para a constante dielétrica que, conforme [18, 19], pode ser grande na presença de acumulações de água e pode ser pequeno em ambiente de reservatório de petróleo.

Empregamos três ordens de regularização: zero, um e dois e o parâmetro ótimo selecionado deve estar associado ao mínimo de cada curva da função GCV. Geramos curvas da função GCV em relação ao parâmetro $\lambda$ como variável independente para cada ordem de regularização. Como forma de quantificar a precisão da inversão e também como critério adicional na seleção do fator $\lambda$, o erro RMS relativo foi calculado. Entende-se o erro RMS entre os dados como erro entre os tempos de trânsito observados e calculados segundo a equação:

$$
E_{\mathbf{d}, r m s}=\frac{\sqrt{\sum_{i=1}^{M}\left(t_{i}^{c a l}-t_{i}^{o b s}\right)^{2}}}{\sqrt{\sum_{i=1}^{M}\left(t_{i}^{o b s}\right)^{2}}} \times 100 \%,
$$

e o erro RMS da velocidade estimada como erro entre a velocidade verdadeira e estimada segundo a equação:

$$
E_{\mathbf{v}, r m s}=\frac{\sqrt{\sum_{i=1}^{N}\left(v_{i}^{\text {est }}-v_{i}^{v e r}\right)^{2}}}{\sqrt{\sum_{i=1}^{N}\left(v_{i}^{v e r}\right)^{2}}} \times 100 \%,
$$

este critério tem finalidade de validar o método de seleção de $\lambda$, pois possibilita conhecer o melhor modelo estimado. Na primeira iteração partiu-se de um modelo inicial de velocidade eletromagnética constante igual a $150 \mathrm{~m} / \mu \mathrm{s}$ equivalendo ao traçado de raios retos.

Para a regularização de ordem 0, a Figura (2a) mostra as curvas GCV para as 4 iterações realizadas mostrando um $\lambda$ ótimo associado ao mínimo em cada iteração. A Figura (3a) mostra a recuperação tomográfica para a primeira iteração e a Figura (3b) para a quarta iteração. Verificou-se convergência e o critério de parada foi considerado quando a variação nos valores de velocidades tornou-se mínima. No caso da regularização de ordem 1, a Figura (2b) mostra as curvas GCV para as 4 iterações realizadas mostrando um $\lambda$ ótimo associado ao mínimo em cada iteração.

As Figuras (4a) e (4b) mostram a recuperação tomográfica equivalente. Tal qual o caso anterior verificou-se convergência para a solução e que 4 iterações são suficientes. Finalmente, para a regularização de ordem 2 , a Figura (2r) mostra as curvas GCV para as 4 iterações realizadas mostrando um $\lambda$ ótimo associado ao mínimo em cada iteração.

As Figuras (5a) e (5b) mostram a recuperação tomográfica na iteração 1 e 4 . Mais uma vez a solução considerada estável foi a quarta iteração. Podemos notar que as imagens obtidas nas iterações finais são mais apuradas, em particular mais interfaces são reveladas, assim como as camadas estão melhor definidas ou resolvidas. Um sumário dos resultados pode ser encontrado na Tabela (I).

\section{CONCLUSÕES}

Neste trabalho avaliou-se o critério GCV 


\begin{tabular}{|c|c|c|c|c|}
\hline \multicolumn{5}{|c|}{ Relação dos Resultados Obtidos } \\
\hline Iteração & $\mathrm{D}_{n}$ & $\lambda$ ótimo & $\mathrm{E}_{\mathbf{d}, r m s}(\%)$ & $\mathrm{E}_{\mathbf{v}, r m s}(\%)$ \\
\hline \multirow{3}{*}{1} & 0 & $10^{2}$ & 0,004 & 0,13 \\
\cline { 2 - 5 } & 1 & $10^{3}$ & 0,44 & 13,62 \\
\cline { 2 - 5 } & 2 & $10^{3}$ & 0,46 & 13,94 \\
\hline \hline \multirow{3}{*}{2} & 0 & $10^{3}$ & 0,004 & 0,044 \\
\cline { 2 - 5 } & 1 & $10^{3}$ & 0,37 & 5,1 \\
\cline { 2 - 5 } & 2 & $10^{2}$ & 0,32 & 6,88 \\
\hline \hline \multirow{3}{*}{3} & 0 & $10^{4}$ & 0,004 & 0,006 \\
\cline { 2 - 5 } & 1 & $10^{4}$ & 0,33 & 0,87 \\
\cline { 2 - 5 } & 2 & $10^{2}$ & 0,25 & 4,42 \\
\hline \hline \multirow{3}{*}{4} & 0 & $10^{4}$ & 0,003 & 0,0035 \\
\cline { 2 - 5 } & 1 & $10^{5}$ & 0,35 & 0,22 \\
\cline { 2 - 5 } & 2 & $10^{3}$ & 0,36 & 3,40 \\
\hline
\end{tabular}

TABELA I: Resultados obtidos para a inversão tomográfica com traçado de raios curvos, utilizando matrizes de derivadas de ordem $0,1 \mathrm{e}$ 2 e o método GCV para obtenção do $\lambda$ ótimo de regularização.

para a escolha do fator ótimo de regularização em tomografia eletromagnética interpoços com um modelo sintético. As inversões tomográficas delimitaram as interfaces adequadamente e possibilitaram a visualizção da zona de acumulação de petróleo.

O critério GCV mostrou-se conveniente para estimativa do $\lambda$ ótimo na inversão linearizada, assim como a regularização e traçado de raios mostraram-se adequados ao problema proposto. Nota-se nos tomogramas estimados que as regularizações de ordem um e dois forneceram melhores resultados, pois a suavização é efetiva com os operados de derivadas.

As simulações permitem concluir que para se adquirir bons resultados é requerido qualidade dos dados de entrada além da cobertura, iluminação do meio pelos raios.

\section{Agradecimentos}

N.P. Oliveira agradece à Fapesb e ao $C N P q$ pelas bolsas de iniciação científica e à $A N P$ pela bolsa de graduação. A. Bassrei agradece ao $C N P q$ pela bolsa de produtividade de pesquisa. Os autores agradecem ao $C N P q$ e Petrobras
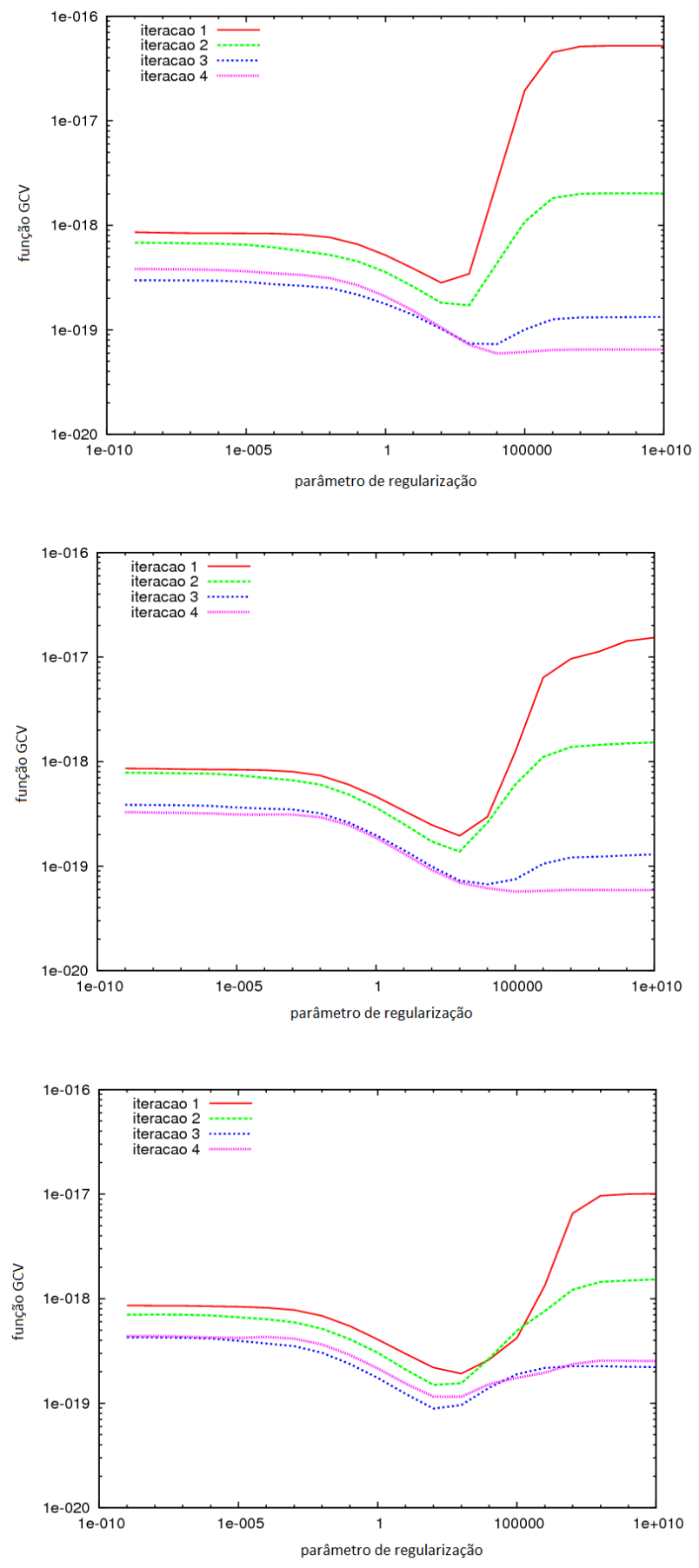

FIGURA 2: Curvas GCV em função do parâmetro de regularização, para diferentes iterações de traçado de raio, para regularização de (a) ordem 0; (b) ordem 1; (c) ordem 2.

pelo apoio ao Instituto Nacional de Ciência e Tecnologia de Geofísica de Petróleo (INCTGP), à Finep pelo apoio à Rede Coopera- 
tiva de Pesquisa em Geofísica de Exploração (Rede 01) e à Petrobras pelo apoio ao projeto "Investigação do Uso da Tomografia In- terpoços como Ferramenta para Caracterização de Reservatórios Complexos."
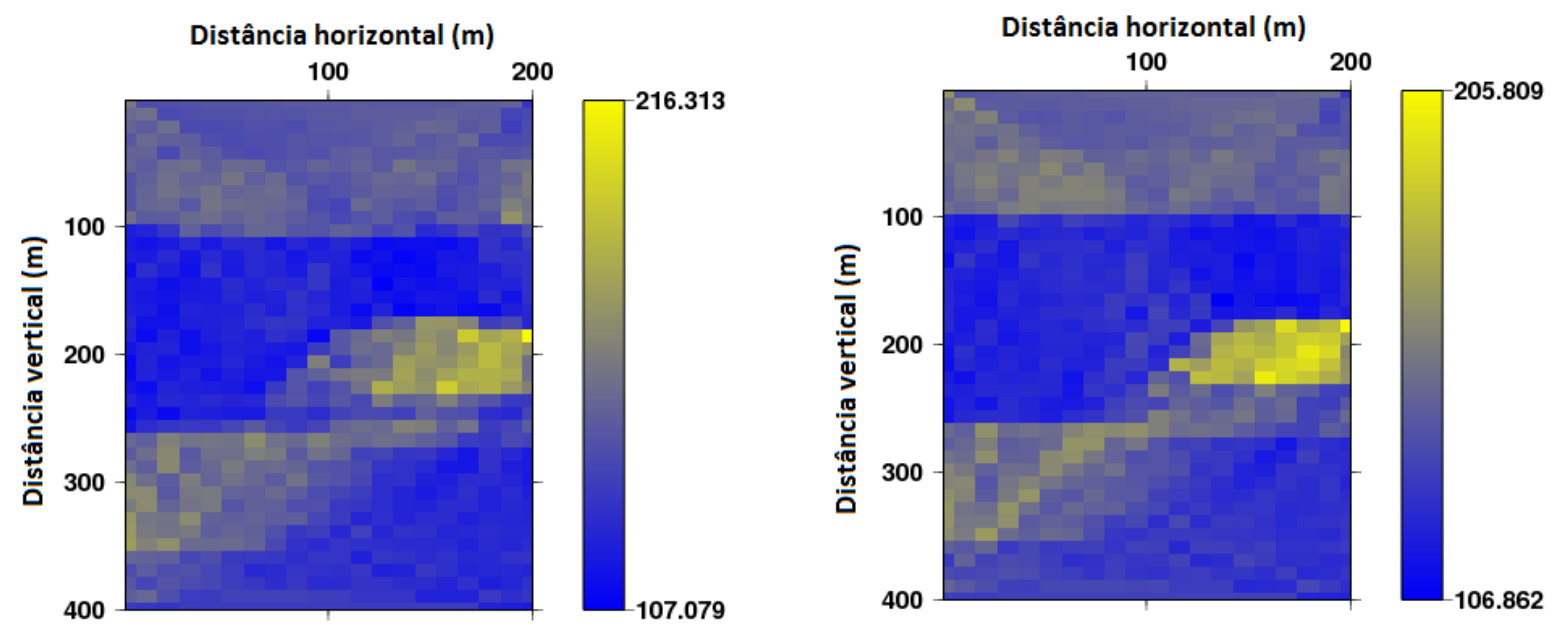

FIGURA 3: Tomograma estimado para regularização de ordem 0, obtida na (a) primeira iteração; (b) quarta iteração.
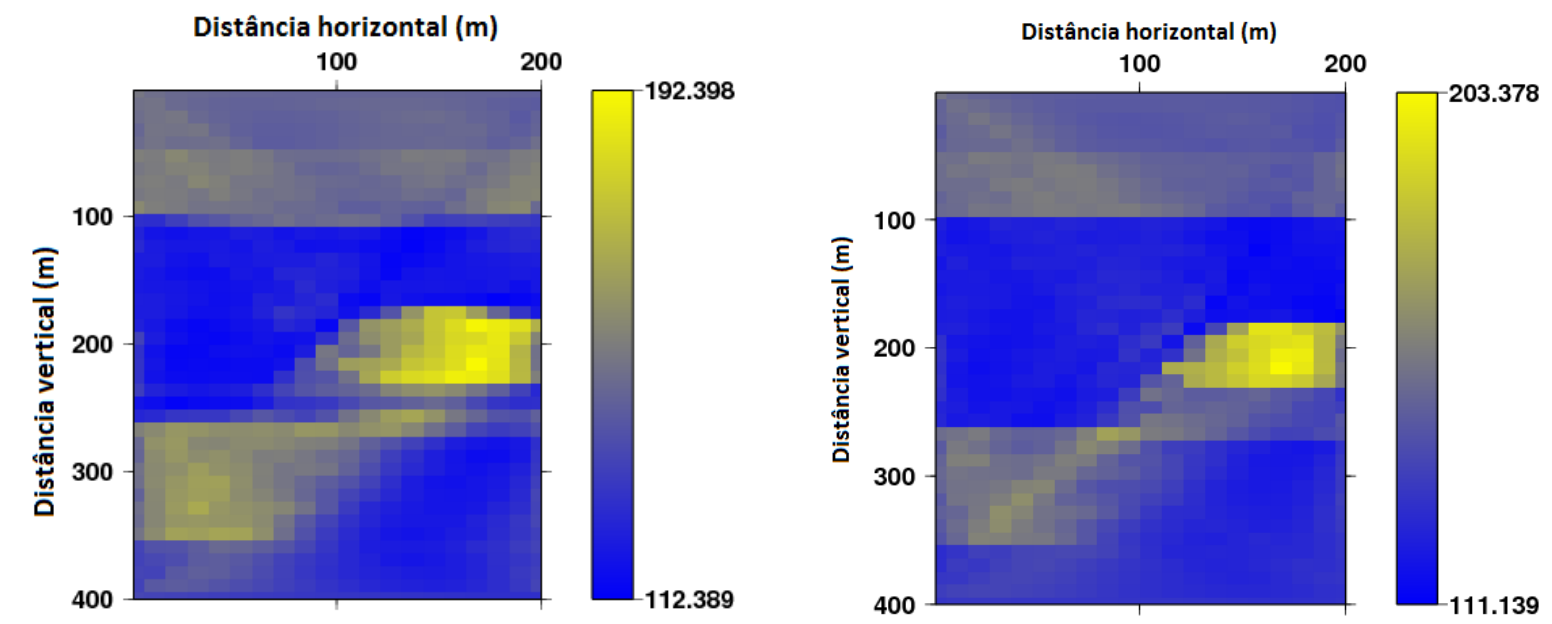

FIGURA 4: Tomograma estimado para regularização de ordem 1, obtida na (a) primeira iteração; (b) quarta iteração. 

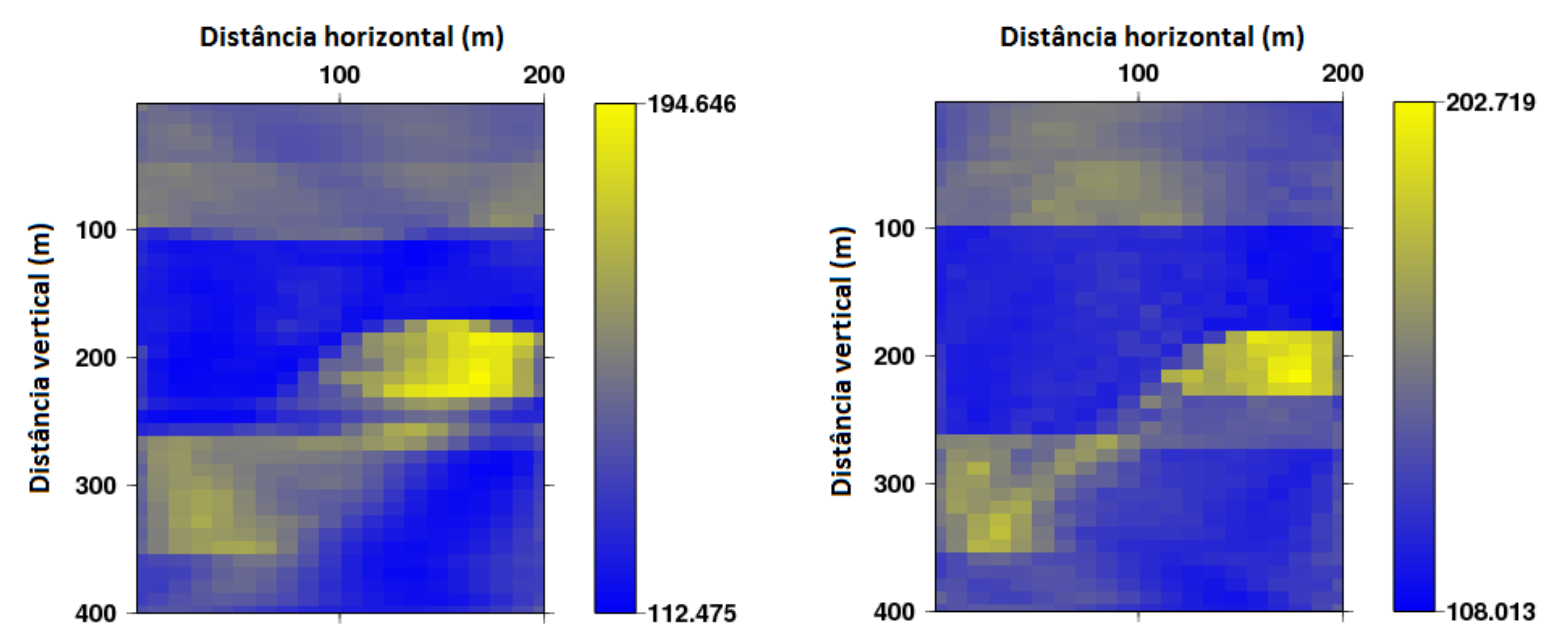

FIGURA 5: Tomograma estimado para regularização de ordem 2, obtida na (a) primeira iteração; (b) quarta iteração.

[1] W. Menke, Geophysical Data Analysis: Discrete Inverse Theory. San Diego: Academic Press (1989).

[2] J.N.P. Silva, A. Bassrei, Critérios de seleção de valores singulares em problemas inversos lineares: uma aplicação em tomografia de tempos de trânsito. Sitientibus Série Ciências Físicas 3, 32 (2007).

[3] A. Bassrei, T. Lemaire, Three-dimensional reconstruction of non-homogeneous dielectric objects by the coupled-dipole method. Braz. J. Phys. 37, 325 (2007).

[4] A. Bassrei, W. Rodi, Regularization and inversion of linear geophysical data. In: International Congress of the Brazilian Geophysical Society, 3rd, 1, 111 1993. Rio de Janeiro (1993).

[5] E.F. Santos, A. Bassrei, J. Costa, Evaluation of L-Curve and and $\Theta$-Curve approaches for the selection of regularization parameters in anisotropic traveltime tomography. J. Seismic Exploration 15, 245 (2006).

[6] E.T.F. Santos, A. Bassrei, L-Curve and $\Theta$ Curve for the selection of regularization parameter in geophysical diffraction tomography. Computers \& Geosciences 33, 618 (2007).

[7] F. Terra, A. Bassrei, E.T.F. Santos, Metodologias de extração do parâmetro ótimo de regu- larização a partir da curva L: aplicação em tomografia de tempos de trânsito. In: 10th International Congress of the Brazilian Geophysical Society (2007).

[8] E.T.F. Santos, J. Harris, A. Bassrei, J. Costa, Trigonal meshes in diffraction tomography with optimum regularization: an application for carbon sequestration monitoring. J. Seismic Exploration 18, 135(2009).

[9] G. Wahba, Spline Models for Observational Data. Filadelfia: SIAM (1990).

[10] C.G. Farquharson, D.W. Oldenburg, A comparison of automatic techniques for estimating the regularization parameter in non-linear problems. Geophysical Journal International 156, 411 (2004).

[11] E.T.F. Santos, A. Bassrei, Application of $G C V$ in geophysical diffraction tomography. In: European Association of Geoscientists and Engineers Conference \& Exhibition, 69th, 2007. Londres (2007).

[12] C.J.M.G. Silva, Tomografia Sísmica de Difração para Monitoramento da Injeção de $\mathrm{CO}_{2}$ em Reservatórios Salinos. Dissertação (Mestrado) - Instituto de Geociências da UFBA, Salvador (2013).

[13] N.P. Oliveira, A. Bassrei, Critério da Validação Cruzada Generalizada na Seleção do 
Parâmetro Ótimo de Regularização em Tomografia Sísmica Interpoços. In: Simpósio Brasileiro da SBGf, 4 , 2010. Brasília (2010).

[14] R.R. Stewart, Exploration Seismic Tomography: Fundamentals. Tulsa: Society of Exploration Geophysicists (1991).

[15] W.M. Telford, L.P. Geldart, R.E. Sheriff, $A p$ plied Geophysics (2nd Edition). Cambridge: Cambridge University Press (1990).

[16] A.H. Andersen, A.C. Kak, Digital ray tracing in two-dimensional refractive Fields. J. Acoustical Soc. America 72, (5) 1593 (1982).
[17] H.A. Schots, Tomografia Sísmica Poço a Poço e Poço a Superfície Utilizando Ondas Diretas. Dissertação (Mestrado) - Instituto de Geociências da UFBA, Salvador (1990).

[18] A.G. Nekut, Electromagnetic ray-trace tomography. Geophysics 59, 371 (1994).

[19] A.P. Annan, Ground Penetrating Radar Workshop Notes. In: Physical Properties I, Cap. 3, Sensors \& Software Inc.. Ontario (2001). 